\title{
Three Dimensional Laparoscopy Improves Surgical Performance: Comparative Study in a Cadaver
}

Jin Kwon Lee, M.D. ${ }^{1}$, Suk-Hwan Lee, M.D., Ph.D. ${ }^{2}$, Jun Gi Kim, M.D., Ph.D. ${ }^{3}$, Yoon Suk Lee, M.D., Ph.D. ${ }^{4}$, Kil Yeon Lee, M.D., Ph.D. ${ }^{5}$ Sun Jin Park, M.D., Ph.D. ${ }^{5}$, Bong Hyeon Kye, M.D., Ph.D. ${ }^{6}$, Sang Chul Lee, M.D., Ph.D. ${ }^{7}$, Sang Woo Lim, M.D., Ph.D. ${ }^{8}$, SIMPLE Study Group

Department of Surgery, 'Changwon Gyeongsang National University Hospital, Gyeongsang National University, Changwon, ${ }^{2}$ Kyung Hee University Hospital at Gangdong, Kyung Hee University School of Medicine, Seoul, ${ }^{3}$ Seoul St. Mary's Hospital, The Catholic University of Korea, Seoul, ${ }^{4}$ Incheon St. Mary's Hospital, The Catholic University of Korea, Incheon, ${ }^{5}$ Kyung Hee University Medical Center, Kyung Hee University School of Medicine, Seoul, ${ }^{6} S t$. Vincent Hospital, The Catholic University of Korea, Suwon, 'Daejeon St. Mary's Hospital, The Catholic University of Korea, Daejeon, ${ }^{8} \mathrm{Halllym}$ Sacred Heart Hospital, Hallym University College of Medicine, Anyang, Korea

Purpose: Conventional laparoscopy using a two-dimensional (2D) has limited performance because of insufficient representation of the stereoscopic effect. Development of three-dimensional (3D) imaging technology has improved depth perception, shortened the execution time and reduced error number. This study was designed to identify the effects of 3D imaging on surgical performance for skilled professionals and surgical residents.

Methods: Two laparoscopic skills tasks, each with three repetitions, were performed by seven experienced laparoscopic surgeons, two minimally experienced laparoscopic surgeons, and three inexperienced surgical residents under both $2 \mathrm{D}$ and 3D conditions with two cadavers. Outcome measures were time for task completion and subjective assessment of performance.

Results: Suturing was completed by all participants and anchoring with V-Loc was performed by 10 participants. Suturing and anchoring time were significantly shorter with 3D laparoscopic in all participants (suturing time, $p=0.011$; anchoring time, $p=0.005$ ). Significant differences were observed between experienced and minimally experienced surgeons (suture time, $p=0.021$; anchoring time, $p=0.018$ ). There was no significant difference among inexperienced surgical residents, but they preferred 3D imaging over 2D.

Conclusion: 3D laparoscopy is associated with a significantly shorter time for performance by experienced surgeons. Our results suggest that 3D laparoscopy will be helpful for surgeons conducting laparoscopic procedures.

Keywords: 3-D Imaging, Laparoscopy, Cadaver
Received May 27, 2016

Revised June 9, 2016

Accepted June 9, 2016

Corresponding author

Suk-Hwan Lee

Department of Surgery, Kyung Hee University Hospital at Gangdong,

Kyung Hee University School of Medicine, 892 Dongnam-ro, Gangdong-gu, Seoul 05278, Korea Tel: $+82-2-440-6134$

Fax: +82-2-440-6295

E-mail: leeshdr@khu.ac.kr
This is an Open Access article distributed under the terms of the Creative Commons Attribution Non-Commercial License (http:/l creativecommons.org/licenses/by-nc/4.0/) which permits unrestricted non-commercial use, distribution, and reproduction in any medium, provided the original work is properly cited.

\author{
Copyright $\odot 2016$ The Journal of Minimally \\ Invasive Surgery. All rights reserved.
}

\section{INTRODUCTION}

With a recent technical revolution in surgical practice over 25 years since the introduction of laparoscopic surgery, three dimensional (3D) imaging technology has introduced and improved the stereoscopic vision and depth perception. Compared with two dimensional laparoscopic techniques, 3D imaging technique improved the operator's performance of pro- 
cedure like hand-to-eye coordination, intracorporeal suturing, and fine dissection. ${ }^{1}$ This advantage is associated with shorter operation times.

Reasons for an advantage for 3D over a 2D system are improvements of depth perception, spatial location, and precision of surgical performance. ${ }^{2,3}$ However, there are disadvantage for 3D system, the need to wear goggles for the operation team, heavier instruments for 3D. ${ }^{4}$ It remains unclear whether 3D imaging system improves the surgical performance with different results of 2D versus 3D.

Aim of this study is to identify the effect of 3D imaging on surgical performance for skilled professional and surgical resident in the cadaver laboratory.

\section{MATERIALS AND METHODS}

Twelve participants were enrolled in the study, including 7 experienced laparoscopic colorectal surgeon who are members of SIMPLE (SIngle vs MultiPort Laparoscopic colEctomy) study, 2 minimally experienced laparoscopic surgeon, and 3 inexperienced surgical residents.

They asked to complete 2 tasks in two cadavers with same circumference like real operation, using 2D and 3D imaging. Two Olympus VISERA ELITE CLV-S190 system were used for $2 \mathrm{D}$ and $3 \mathrm{D}$ vision with $10-\mathrm{mm}, 30$ degree laparoscope with high-definition video monitor of SONY cooperation. This system can change view modes between 3D and 2D with same laparoscope.

Two laparoscopic skill tasks were intracorporeal suturing and intracorporeal anchoring. In task for intracorporeal suturing, all participants were asked to grasp needle with the needle holder and dissector in the abdominal cavity of cadaver, throw one surgeon's knot square with additional two knots and cut with endo-scissors. This task was repeated three times with each visual system in cadaver. Participants performed the tasks first in 2D, then in 3D. We recorded total time for each task

In task for intracorporeal anchoring, V-Loc Wound Closure Device (Covidien, Inc.) 3-0 needle had to be passed through distal end hole of V-Loc using a needle holder. All partici- pants were asked to grasp needle in the abdominal cavity of cadaver, pass through the hole with needle. We recorded total time for each task.

Outcome measures were time for task completion and subjective assessment of performance Statistical analysis was performed using SPSS statistical software (SPSS Inc., Chicago, IL). $p<.05$ defined statistical significance.

\section{RESULTS}

The participants finished the 2 tasks of intracorporeal suturing as well as anchoring on cadaver.

Intracorporeal suturing time was significantly shorter using 3D imaging in all participants $(p=0.011)$. Experienced and minimally experienced surgeons showed a significantly shorter performance $(p=0.021)$, but inexperienced surgical resident did not show significant statistically difference $(p=0.285)$.

Intracorporeal anchoring time was significantly better using 3D imaging in all participants $(p=0.005)$. Experienced and minimally experienced surgeons took shorter time to perform the task $(p=0.018)$, but inexperienced surgical resident did not improve the performance statistically $(p=0.109)$ (Table 1).

When compared with 2D imaging system, all participants prefer the 3D imaging system. Although surgical residents showed no statistically benefit, they preferred the 3D system because of better depth perception.

\section{DISCUSSION}

Three-dimensional imaging system for laparoscopy provide better visualization for operator, but there are many debates for standardization. Most of the systematic review evaluated the effect of 3D vision on laparoscopic performance revealed a significant shortening in performance time, but one-third could not prove significant statistical difference between 2D and 3D imaging systems. ${ }^{5}$

Recent RCTs reveal more benefits than previous studies because of development of advanced 3D visualization equipment. ${ }^{5}$ Another recent studies showed fewer or no disadvantage compared with previous 3D imaging system because of

Table 1. Total elapse time to performing the task to compare two-dimensional versus three-dimensional imaging system

\begin{tabular}{|c|c|c|c|c|c|c|}
\hline & \multicolumn{2}{|c|}{$\begin{array}{l}\text { Experienced surgeon } \\
\qquad(\mathrm{n}=9)\end{array}$} & \multicolumn{2}{|c|}{$\begin{array}{l}\text { Inexperienced surgical resident } \\
\qquad(\mathrm{n}=3)\end{array}$} & \multicolumn{2}{|c|}{$\begin{array}{l}\text { Total participants } \\
\qquad(n=12)\end{array}$} \\
\hline & $2 \mathrm{D}$ & 30 & $2 \mathrm{D}$ & 30 & $2 \mathrm{D}$ & 30 \\
\hline Intracorporeal suturing time (sec) & $332.1 \pm 63.6$ & $266.7 \pm 35.3^{*}$ & $646.3 \pm 130.7$ & $528.7 \pm 238.5$ & $410.6 \pm 161.9$ & $332.2 \pm 159.0^{*}$ \\
\hline Intracorporeal anchoring & $14.8 \pm 6.9$ & $7.3 \pm 2.5^{*}$ & $16.6 \pm 6.6$ & $10.8 \pm 2.0$ & $15.32 \pm 6.50$ & $8.36 \pm 2.83^{*}$ \\
\hline
\end{tabular}

$2 \mathrm{D}=$ two-dimensional; $3 \mathrm{D}=$ three dimensional. ${ }^{*} p<.05$ for $3 \mathrm{D}$ versus $2 \mathrm{D}$. 
improved image quality.

The most influenced advantage of using 3D imaging system is advanced depth perception, which facilitate to reduce the cognitive workload and improve surgical performance and let down rate of errors and improve the quality of laparoscopic surgery and patient safety by reducing the visual misperception. ${ }^{4,67}$

Storz et al. showed that experienced surgeons did not gain time advantage in a 3D imaging system, but obtained overall precision with 3D visualization. ${ }^{8}$ Another studies showed that experienced laparoscopic surgeons learned to manage the loss of depth perception with more extensive experience with 2D imaging system, thus there was no benefits using 3D imaging system in experienced surgeons. ${ }^{2,9}$ This study suggests that $3 \mathrm{D}$ imaging system provides the advantages in surgical performance, especially experienced surgeon who had experienced many laparoscopic surgery with 2D imaging system. During the suturing and anchoring, time for grasping the needle and thread was significantly reduced. The improvement on visual depth perception may reduce the misperception of participants and time for performing the task..$^{10}$ Because we did not distinguish the time and precision of performance, there was opposite result compared previous study. However, experienced surgeons in our study said 3D imaging system may enable to perform precise procedure during the task like grasping the needle and thread. We suppose that fine precise performance enable to reduce overall time for task in 3D imaging system.

Kong et all revealed that inexperience group had no difference in time to complete the task. ${ }^{11}$ For reducing the time to perform the task, repeated training is more needed with advanced performance skills.

Although 3D imaging system is developed rapidly, some side effect of 3D imaging system still remained like headaches, dizziness, eye strain, and physical discomfort. ${ }^{12,13}$ This disadvantage has been supplemented by the more advanced visual systems. In this study, two surgeon felt dizziness and fatigue of eyes. Another disadvantage of 3D imaging system is still more expensive equipments. ${ }^{6}$

Compared with Robot-assisted systems, 3D imaging systems may be used for many routine procedure in benign and malignant condition with a more relevant option by providing 3D vision. However robot-assisted systems can perform more precise and accurate procedure by combination of articulated instruments with 3D imaging system. ${ }^{6}$

This study was conducted with members of SIMPLE (SIngle vs MultiPort Laparoscopic colEctomy) study on cadaver workshop. SIMPLE study group were made up with experts in laparoscopic colorectal surgery in Korea. Prospective randomized trial for benefit and effectiveness of single port laparoscopic surgery in colorectal cancer surgery with long-term oncologic result is in progress with SIMPLE study group.

Limitations of our study are too small participants for evaluate the efficacy of 3D imaging system and lack of tasks verifying the effects of 3D. More participants and more reliable investigation tool and tasks for verifying the effect of 3D imaging system are needed in future studies.

In conclusion, three-dimensional laparoscopy seems to reduce significantly time for performance with fine precision when compared to 2D laparoscopy. The advantages were more significant in experienced surgeons than inexperienced surgical residents. For standardization of 3D laparoscopy, more variable studies is needed.

\section{REFERENCES}

1) Tuschy B, Berlit S, Brade J, Sutterlin M, Hornemann A. Full Highdefinition three-dimensional gynaecological laparoscopy--clinical assessment of a new robot-assisted device. In Vivo 2014;28:111115.

2) Cicione A, Autorino R, Breda A, et al. Three-dimensional vs standard laparoscopy: comparative assessment using a validated program for laparoscopic urologic skills. Urology 2013;82:14441450.

3) Lusch A, Bucur PL, Menhadji AD, et al. Evaluation of the impact of three-dimensional vision on laparoscopic performance. J Endourol 2014;28:261-266.

4) Wilhelm D, Reiser S, Kohn N, et al. Comparative evaluation of HD 2D/3D laparoscopic monitors and benchmarking to a theoretically ideal 3D pseudodisplay: even well-experienced laparoscopists perform better with 3D. Surg Endosc 2014;28:2387-2397.

5) Sorensen SM, Savran MM, Konge L, Bjerrum F. Three-dimensional versus two-dimensional vision in laparoscopy: a systematic review. Surg Endosc 2016;30:11-23.

6) Smith R, Schwab K, Day A, et al. Effect of passive polarizing three-dimensional displays on surgical performance for experienced laparoscopic surgeons. Br J Surg 2014;101:1453-1459.

7) Tanagho YS, Bhayani SB, Sandhu GS, Vaughn NP, Nepple KG, Figenshau RS. Renal functional and perioperative outcomes of off-clamp versus clamped robot-assisted partial nephrectomy: matched cohort study. Urology 2012;80:838-843.

8) Storz P, Buess GF, Kunert W, Kirschniak A. 3D HD versus 2D HD: surgical task efficiency in standardised phantom tasks. Surg Endosc 2012;26:1454-1460.

9) Patel HR, Ribal MJ, Arya M, Nauth-Misir R, Joseph JV. Is it worth revisiting laparoscopic three-dimensional visualization? A validated assessment. Urology 2007;70:47-49.

10) Alaraimi B, El Bakbak W, Sarker S, et al. A randomized prospective study comparing acquisition of laparoscopic skills in threedimensional (3D) vs. two-dimensional (2D) laparoscopy. World J Surg 2014;38:2746-2752. 
11) Kong SH, Oh BM, Yoon H, et al. Comparison of two- and threedimensional camera systems in laparoscopic performance: a novel 3D system with one camera. Surg Endosc 2010;24:1132-1143.

12) Chan AC, Chung SC, Yim AP, Lau JY, Ng EK, Li AK. Comparison of two-dimensional vs three-dimensional camera systems in laparoscopic surgery. Surg Endosc 1997;11:438-440.

13) Mueller MD, Camartin C, Dreher E, Hanggi W. Three-dimensional laparoscopy. Gadget or progress? A randomized trial on the efficacy of three-dimensional laparoscopy. Surg Endosc 1999;13:469-472. 